\title{
Aicardi Syndrome Associated with Autosomal Genomic Imbalance: Coincidence or Evidence for Autosomal Inheritance with Sex-Limited Expression?
}

\author{
P. Prontera ${ }^{a}$ d $\quad$ A. Bartocci ${ }^{b} \quad$ V. Ottaviani ${ }^{a} \quad$ I. Isidoria $\quad$ D. Rogaia ${ }^{a} \quad$ C. Ardisia ${ }^{a}$ \\ G. Guercinic ${ }^{c}$ A. Mencarelli ${ }^{a} \quad$ E. Donti ${ }^{a}$ \\ ${ }^{a}$ Medical Genetics Unit, University of Perugia, ${ }^{b}$ Neurophysiopathology and ${ }^{\mathrm{C}}$ Neuroradiology, Hospital S. Maria della \\ Misericordia, and ${ }^{\mathrm{d} D e p a r t m e n t}$ of Experimental Medicine and Biochemical Sciences, University of Perugia, \\ Perugia, Italy
}

\section{Key Words}

Aicardi syndrome - Autosomal inheritance - Genomic

disorder $\cdot$ Sex-limited expression

\begin{abstract}
Aicardi syndrome (AIS), a rare neurodevelopmental disorder thought to be caused by an X-linked dominant mutation, is characterized by 3 main features: agenesis of corpus callosum, infantile spams and chorioretinal lacunae. A genomewide study of a girl with AIS lead us to identify a $6 q$ deletion; $12 q$ duplication, derived from a maternal $6 q ; 12 q$ translocation. The two intellectually impaired brothers of the proband showed the same genomic anomalies, but not the constellation of features characterizing the AIS. This could be either a coincidental observation of 2 rare conditions, but can also suggest an alternative hypothesis for the genetic etiology of AIS, indicating the existence of a subset of autosomal genes whose mutation could act in a sex-confined manner.

Copyright $\odot 2013$ S. Karger AG, Basel
\end{abstract}

(c) 2013 S. Karger AG, Basel

1661-8769/13/0044-0197\$38.00/0
Aicardi syndrome (AIS, OMIM 304050), identified by Dr. Jean Aicardi [Aicardi et al., 1965], is a rare neurodevelopmental disorder that affects almost exclusively females, defined by the triad of partial or complete absence of the corpus callosum, infantile spams and typical chorioretinal lacunae. Revised criteria include: characteristic facial features, such as prominent premaxilla, upturned nasal tip, decreased angle of the nasal bridge, sparse lateral eyebrows, coloboma of the optic nerve, in addition to the chorioretinal lacunae, microcephaly, periventricular heterotopias, microgyria, enlarged ventricles or porencephalic cysts. Moreover, moderate-to-severe developmental delay and intellectual disability is found in almost all patients [Aicardi et al., 1965; Bertoni et al., 1979; Aicardi, 1999, 2005; Roser, 2003; Sutton et al., 2005; Hopkins et al., 2008].

The etiology of AIS is still unknown, even if a genetic etiology due to a de novo X-linked dominant mutation lethal in hemizygous male embryos is strongly suspected [Wettke-Schäfer and Kanter, 1983; Donnenfeld et al.,

\section{KARGER}

E-Mail karger@karger.com

www.karger.com/msy
Paolo Prontera, $\mathrm{PhD}$

Medical Genetics Unit, University of Perugia

Via E. dal Pozzo

IT-06123 Perugia (Italy)

E-Mail paolo.prontera@ ospedale.perugia.it 

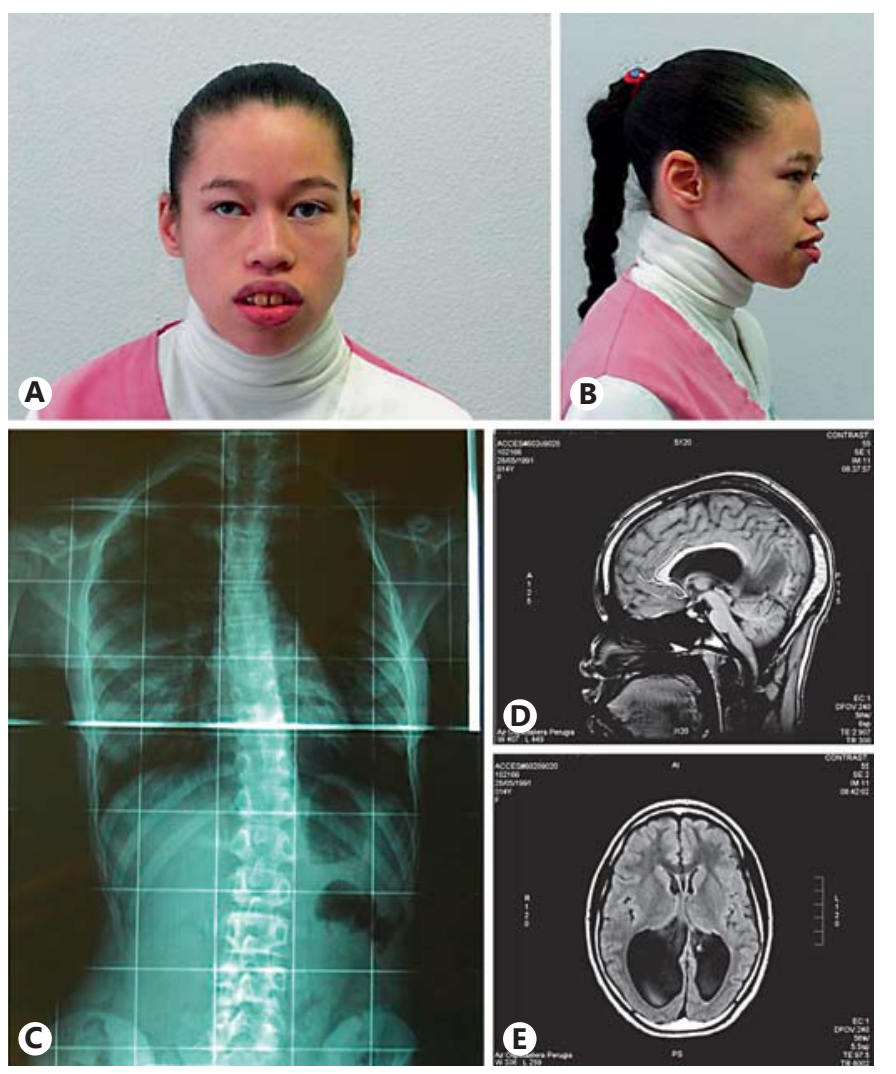

Fig. 1. A, B Note the facial dysmorphisms of the patient, in particular the strabismus, high forehead, fullness of periorbital region, prominent premaxilla, prognatism, short philtrum, open-mouth appearance, long mandible, thick lips. C Anterior-posterior X-ray projection of the vertebral column showing the moderate scoliosis. The MRIs show the partial agenesis of corpus callosum (D) and ventriculomegaly with gross cerebral asymmetry (E).

1989, 1990; Van der Veyver, 2002; Rosser, 2003; Aicardi, 2005]. About 200 girls with AIS have been reported in the literature [Donnenfeld et al., 1989; Rosser, 2003; Aicardi, 2005; Glasmacher et al., 2007; Grosso et al., 2007]. Chromosome and genomic (e.g. array-CGH) studies of AIS patients are normal in the majority of the cases with only few exceptions [Ropers et al., 1982; Naritomi et al., 1992; Bursztejn et al., 2009; Wang et al., 2009]. Ropers et al. [1982] reported a presumably balanced X;3 translocation in a girl with features of AIS, indicating gene or genes located within the Xp22.2p22.3 region as possible candidates. Bursztejn et al. [2009] reported an 8-year-old girl with an initial diagnosis of AIS who subsequently found to carry a de novo $11.73-\mathrm{Mb}$ terminal deletion of $1 \mathrm{p} 36$ chromosome band and emphasized the phenotypic overlap between the 2 disorders.
In this study, we report a sibship of a girl with AIS and her 2 intellectually impaired brothers, all having the same 6q deletion;12q duplication, derived from the malsegregation of a maternal balanced translocation.

\section{Clinical Report}

\section{Clinical History}

Personal and familial histories of the parents were unremarkable. The propositus, a 21 -year-old girl, had 3 brothers of 16,8 and 6 years, respectively. She was born at 39 weeks gestation after a dystocial delivery, weighting 3,550 g. She showed global neurodevelopmental delay and was able to walk alone and use a few words at 2 years of age. The mother referred that from the fourth to the sixth month and then later on, at 2 years of age, she showed episodes of spasms, mainly characterized by rigidity with stiffening of the arms and legs. At 4 years of age, she developed an epileptic partial crisis, characterized by head and eye rotation, preceeded by vomiting, nausea and cyanosis and followed by unconsciousness. The EEG showed multifocal, parossistic alteration, bioelectric activity disorganization, but not hypsarrhythmia. Similar EEG findings were recorded during the first and second decades of life, with a crises frequency of 3-4/month. The epilepsy was resistant to pharmacologic treatment until the introduction, 6 years ago, of oxcarbazepine, lamotrigin and sodium valproate which led to a reduction of the crises frequency. The most recent EEG, performed at 20 years of age, revealed slow and parossistic temporo-occipital alteration, mainly in the right hemisphere, always associated with general bioelectric activity disorganization. The MRI disclosed partial corpus callosum agenesis, mainly of the splenium, colpocephaly, ventriculomegaly, gross cerebral asymmetry, periventricular cerebral atrophy, and right temporal lobe cortical dysplasia (fig. 1D, E).

Previous ophthalmologic evaluation, performed at 4 years, disclosed a congenital coloboma of the right optic nerve and yellowwhite, well-circumscribed, depigmented areas of the retinal pigment epithelium (chorioretinal lacunae). The visual acuity was $3 / 10$ in the right eye and 1/60 in the left. At present, the left eye is completely blind. She also developed a severe scoliosis, confirmed by X-ray examination (fig. 1C). The neuropsychological evaluation with the LEITER-R test, at 14 years of age, ascertained a moderate intellectual disability.

The 20-year-old patient presented in our Medical Genetics Unit for the first time. The dysmorphological assessment revealed: height $164 \mathrm{~cm}$ (50th centile), weight $45 \mathrm{~kg}$ (10th centile) and OFC $52 \mathrm{~cm}$ (3th centile), microcephaly, prominent premaxilla, prognatism, short philtrum, open-mouth appearance, long mandible, thick lips, highly arched palate, scoliosis, and proximally placed thumbs (fig. 1A, B).

Neuropsychological assessment with LEITER-R test disclosed mild intellectual delay in 2 out of the 3 brothers; however, their clinical history was very different from that of the sister. In fact, they did not show infantile spasms, visual defects (normal ophthalmologic evaluations), scoliosis, or facial dysmorphisms, and the brain MRI revealed that they both displayed a mild ventricular dilation, but no corpus callosum agenesis or other structural brain defects. 
Fig. 2. Results of the array-CGH analysis (CytoChip ISCA $4 \mathrm{x} 44 \mathrm{~K}$ v1.0 BlueGnome) showing the $6 \mathrm{q} 27$ deletion (A) and the 12q24.32q24.33 duplication (B). FISH on metaphase cells: $\mathbf{C}$ subtelomere specific 6qtel probe (green) and subtelomere specific 6ptel control probe (red) showing the $6 \mathrm{q}$ deletion. D Subtelomere specific 12qtel probe (green) and $\alpha$-satellite chromosome 6 probe (red) as control, showing the $12 \mathrm{q}$ duplication on chromosome 6.

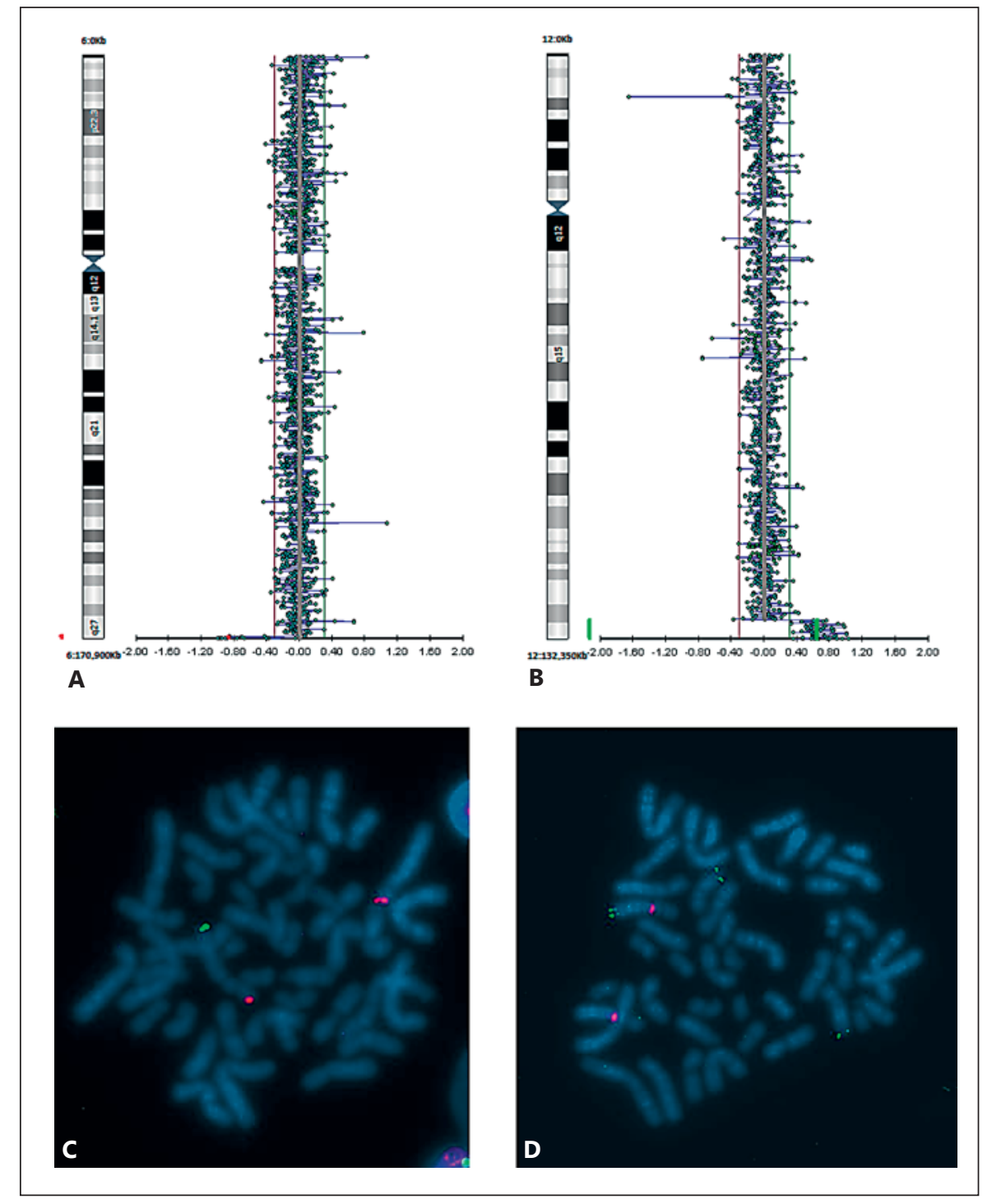

\section{Genetic Testing}

Array-CGH analyses of the proband and her intellectually impaired brothers were performed on DNA extracted from whole blood (PerfectPure DNA Blood Kit, 5Prime GmbH, Hamburg, Germany), using commercially available arrays (CytoChip ISCA $4 \mathrm{x} 44 \mathrm{~K}$ v1.0 BlueGnome, Cambridge, UK) according to the manufacturer's instructions as previously described [Prontera et al., 2009].

The array-CGH revealed the presence of a $\sim 550-\mathrm{kb}$ deletion in the $6 \mathrm{q} 27$ region and a $\sim 4.2-\mathrm{Mb}$ duplication in the $12 \mathrm{q} 24.32 \mathrm{q} 24.33$ region [arr 6q27(170.244.093×2,170.330.025-170.921.059×1).arr 12 q24.32q24.33(129.407.053×2,129.491.848-133.767.956×3)] (Ensembl version 54, Genome build NCBI 37, hg19) in all subjects (fig. 2A).

Fluorescence in situ hybridization (FISH) was performed, as previously reported [Prontera et al., 2009], on chromosome metaphases using subtelomere specific 6qtel probe (clone 57H24), subtelomere specific 12 qtel probe (clone $221 \mathrm{~K} 18$ ) and, as controls, $\alpha$-satellite chromosome 6 probe (locus D6Z1) and subtelomere specific 6ptel probe (clone 62I11) (Aquarius CytoCell, Cambridge, UK) (fig. 2B). Multiple ligation probe amplification (MLPA) (SALSA MLPA kit P036-E1 Human Telomere-3 probemix; SALSA MLPA kit P070-B2 Human Telomere-5; SALSA MLPA kit P277-B1 Human Telomere-10; SALSA MLPA kit P286-B1 Human Telomere-11 - MRC-Holland, Amsterdam) was carried out on DNA extracted from peripheral blood, following the manufacturer's protocols, to better delineate the boundaries of the rearrangements (data not shown). Taken together, the array-CGH and MLPA results permitted establishing that, in all subjects, the last genes deleted on chromosome $6 \mathrm{q}$ and duplicated on chromosome $12 \mathrm{q}$ were DLL1 and GLT1D1, respectively, while the first genes not deleted on chromosome $6 \mathrm{q}$ and not duplicated on chromosome 12q were C6ORF208 and SLC15A4, respectively. There are no other genes mapped between the first 
Table 1. Genes involved in the deletion and duplication

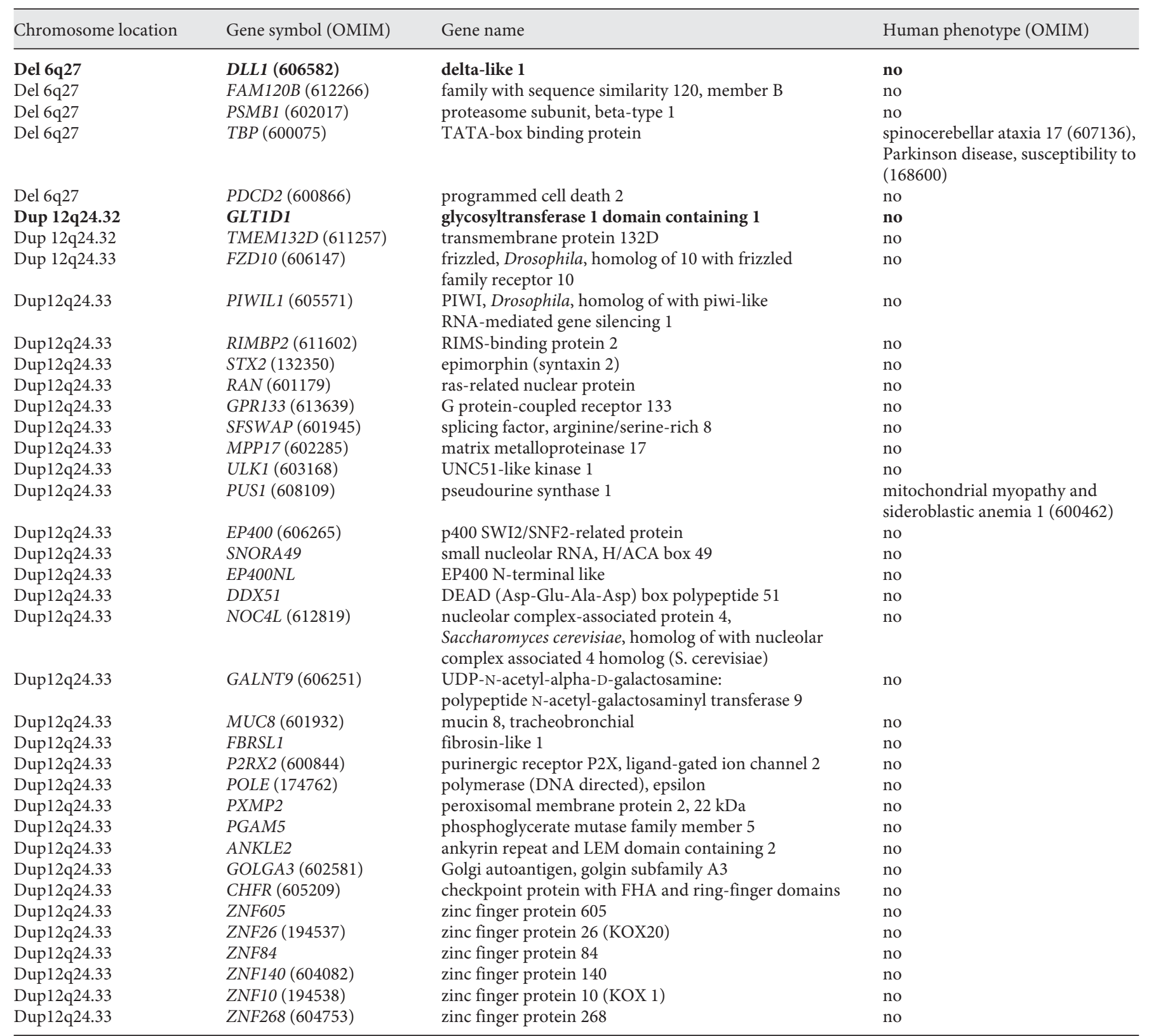

The first deleted and duplicated genes are shown in bold type. The last column shows the associated human phenotype.

deleted or duplicated and the first normal gene. The complete list of the genes deleted and duplicated in our patients are shown in table 1 .

Cytogenetics and FISH analyses performed using the subtelomere specific probes for the chromosomes 6 and 12 terminal long arms, disclosed the presence of an apparently balanced reciprocal $6 / 12$ translocation $[\mathrm{t}(6 ; 12)(\mathrm{q} 27 ; \mathrm{q} 24.33)]$ in the mother and normal karyotypes in the father and healthy brother.

\section{Discussion}

Chromosomal and genomic rearrangements are important findings when encountered in patients who show phenotypic overlap with, presumably, monogenic syndrome of still unknown etiology. In this study analyzing AIS patients, it is hypothetically expected to find $\mathrm{X}$ chromosome abnormalities even if the possibility remains 
that AIS could be caused by a new mutation on an autosome with gender-limited expression.

To the best of our knowledge, there are no cases in the literature of patients with definitive AIS diagnosis and an autosomal genomic imbalance. The patient reported by Bursztejn et al. [2009], with suspected AIS and 1p36 deletion, does not show the chorioretinal lacunae which is a salient feature of the syndrome, being present in about $90-100 \%$ of cases [King et al., 1998; Aicardi, 2005]. Therefore, the authors concluded a possible phenocopy.

The female patient reported here, not only meets the AIS diagnostic criteria, but also has 2 male brothers with the same autosomal genomic imbalance, which show a clinical phenotype similar to each other, but completely different from that of the sister.

Various hypotheses can be proposed to explain this intrafamilial variability: (a) the dosage effect (gain and/or loss) of a gene, or gene subsets, acts in a sex-confined manner leading to AIS in the female and a different phenotype in males; (b) the deletion has uncovered a recessive allele only in the female or the recessiveness acts in a sex-confined manner; (c) the genomic imbalance generated an AIS phenocopy, and (d) the female patient harbors a single gene mutation, which is unknown (leading to AIS), and the genomic imbalance is merely a coincidence. The hypothesis of a recessive allele is in contrast with the inheritance model of AIS, while the hypothesis of a coincidence of 2 such rare conditions (genomic imbalance and single gene de novo mutation) is possible, even if statistically unlikely.

We did not find reports of patients with the same complex rearrangement involving chromosome 6 and 12 in the literature, so that it is not possible to compare our case with previous data. Moreover, patients with an exclusive deletion $6 \mathrm{q}$ or duplication of $12 \mathrm{q}$ are very rare, being in the majority of cases associated with a second rearrangement. The few patients reported with terminal deletion of 6q27 displayed facial dysmorphisms, intellectual disabili- ties, autistic behavior, hirsutism, and general abnormalities of the vision (myopia), while those with similar 12q24.32 duplication showed facial dysmorphisms, intellectual disabilities, hydrocephalus, heart and kidney defects (Ecaruca database at http://umcecaruca01.extern. umcn.nl:8080/ecaruca/ecaruca.jsp; Decipher database at http://decipher.sanger.ac.uk/). The very few cases described are not comparable with our study at a molecular level, in order to state that these genomic regions are not associated with the AIS phenotype. Obviously, we cannot exclude that our complex and unique rearrangement results in a phenocopy; however, why such a wide phenotypic variability exists among female and males patients remains an unanswered question. In this study, we hypothesized that the dosage alteration of gene(s) in the $6 \mathrm{q} / 12 \mathrm{q}$ regions have had a female-limited expression and that the same gene(s) are potential candidates for AIS (table 1).

In conclusion, even if genetic heterogeneity is possible in the AIS, and the 2 proposed inheritance models (Xlinked dominant de novo and autosomal de novo with sex-limited expression) are not mutually excludable, we believe that the extensive molecular analysis of the genes depicted in table 1 could provide new insight into the still unknown etiology of the syndrome. Finally, our findings advise clinicians to perform genome-wide analysis of patients with clinical diagnosis of AIS, since the evidence of a genomic imbalance could change the patient and family management following the diagnosis drastically.

\section{Acknowledgements}

We are indebted to the patients and their family for their participation in our study. We also wish to thank Dr. Rita Romani (Department of Experimental Medicine and Biochemical Sciences, University of Perugia, Perugia, Italy) for her suggestions and helpful comments.

\section{References}

Aicardi Syndrome: The Autosomal

Hypothesis
Aicardi J: Aicardi syndrome: old and new findings. Internat Pediatr 14:5-8 (1999).

Aicardi J: Aicardi syndrome. Brain Dev 27:164171 (2005).

Aicardi J, Levebre J, Lerique-Koechlin A: A new syndrome: spasms in flexion, callosal agenesis, ocular abnormalities. Electroencephalogr Clin Neurophysiol 19:609-610 (1965).

- Bertoni JM, von Loh S, Allen RJ: The Aicardi syndrome: report of 4 cases and review of the literature. Ann Neurol 5:475-482 (1979).
Bursztejn AC, Bronner M, Peudenier S, Grégoire MJ, Jonveaux P, Nemos C: Molecular characterization of a monosomy $1 \mathrm{p} 36$ presenting as an Aicardi syndrome phenocopy. Am J Med Genet A 149A:2493-2500 (2009).

Donnenfeld AE, Packer RJ, Zackai EH, Chee CM, Sellinger B, Emanuel BS: Clinical, cytogenetic, and pedigree findings in 18 cases of Aicardi syndrome. Am J Med Genet 32:461-467 (1989). 
Donnenfeld AE, Graham JM Jr, Packer RJ, Aquino R, Berg SZ, Emanuel BS: Microphthalmia and chorioretinal lesions in a girl with an Xp22.2-pter deletion and partial 3p trisomy: clinical observations relevant to Aicardi syndrome gene localization. Am J Med Genet 37: 182-186 (1990).

- Glasmacher MA, Sutton VR, Hopkins B, Eble T, Lewis RA, et al: Phenotype and management of Aicardi syndrome: new findings from a survey of 69 children. J Child Neurol 22:176184 (2007).

- Grosso S, Lasorella G, Russo A, Galluzzi P, Morgese G, Balestri P: 2007. Aicardi syndrome with favorable outcome: case report and review. Brain Dev 29:443-446 (2007).
Hopkins B, Sutton VR, Lewis RA, Van den Veyver I, Clark GD: Neuroimaging aspects of Aicardi syndrome. Am J Med Genet A 146A:28712878 (2008).

King AM, Bowen DI, Goulding P, Doran RML: Aicardi syndrome. Br J Ophthamol 82:457 (1998).

Naritomi K, Izumikawa Y, Nagataki S, Fukushima Y, Wakui K, et al: Combined Goltz and Aicardi syndromes in a terminal Xp deletion: are they a contiguous gene syndrome? Am J Med Genet 43:839-843 (1992).

- Prontera P, Bernardini L, Stangoni G, Capalbo A, Rogaia D, et al: 2q31.2q32.3 deletion syndrome: report of an adult patient. Am J Med Genet A 149A:706-712 (2009).

- Ropers HH, Zuffardi O, Bianchi E, Tiepolo L: Agenesis of corpus callosum, ocular, and skeletal anomalies (X-linked dominant Aicardi's syndrome) in a girl with balanced $\mathrm{X} / 3$ translocation. Hum Genet 61:364-368 (1982).
Rosser T: Aicardi syndrome. Arch Neurol 60: 1471-1473 (2003).

Sutton VR, Hopkins BJ, Eble TN, Gambhir N, Lewis RA, Van den Veyver IB: Facial and physical features of Aicardi syndrome: infants to teenagers. Am J Med Genet A 138A:254258 (2005).

Van den Veyver IB: Microphthalmia with linear skin defects (MLS), Aicardi, and Goltz syndromes: are they related X-linked dominant male-lethal disorders? Cytogenet Genome Res 99:289-296 (2002).

-Wang X, Sutton VR, Eble TN, Lewis RA, Gunaratne $P$, et al: A genome-wide screen for copy number alterations in Aicardi syndrome. Am J Med Genet A 149A:2113-2121 (2009).

Wettke-Schäfer R, Kantner G: X-linked dominant inherited diseases with lethality in hemizygous male. Hum Genet 64:1-23 (1983). 